\title{
Throughput and Backhaul Energy Efficiency Analysis in Two-Tier HetNets: A MultiObjective Approach
}

\author{
Haris Pervaiz*, Zhengyu Song ${ }^{\dagger}$, Leila Musavian*, Qiang Ni* and Xiaohu Ge ${ }^{\ddagger}$ \\ ${ }^{*}$ School of Computing and Communications, Lancaster University, UK \\ ${ }^{\dagger}$ School of Information and Electronics, Beijing Institute of Technology, China \\ ${ }^{\ddagger}$ School of Electronic Information and Communications, Huazhong University of Science and Technology, China \\ Email: \{h.pervaiz, l.musavian, q.ni\}@lancaster.ac.uk*, z.song912@ gmail.com ${ }^{\dagger}$,xhge@ mail.hust.edu.cn ${ }^{\ddagger}$
}

\begin{abstract}
Fifth Generation (5G) networks will compromise of heterogeneous networks (HetNets) with macrocell overlaid with lower power small cells to achieve higher throughput by offloading users with low signal-to-noise-ratio from macrocell to the small cells. In this paper, we proposed a multi-objective optimization problem (MOP) to jointly investigate the tradeoff between throughput and backhaul energy efficiency (BEE) using $\omega$-fair utility function for two different backhauling technologies in downlink transmission scheme of a two-tier HetNets. We then transform the proposed MOP into a single objective optimization problem (SOP) employing the weighted sum method to obtain the complete Pareto Frontier solution set with minimum QoS requirements and rate fairness level $\omega$. The transformed SOP is solved in an iterative manner using Lagrangian Dual Decomposition (LDD) with a subgradient method providing a near-optimal solution. Simulation results demonstrate the effectiveness of our proposed approach in reducing the total area power consumption irrespective of the backhauling technology by dynamically adjusting weighting coefficient $\alpha$ and rate fairness level $\omega$. Our numerical results also demonstrate the fundamental tradeoff between throughput and BEE for different parameters such as weighting coefficient $\alpha$ and rate fairness level $\omega$.
\end{abstract}

Index Terms-Backhaul Energy efficiency, Green Communication, HetNets, Next Generation Networks, Multi-objective Optimisation Problem.

\section{INTRODUCTION}

$\mathbf{O}$ $\mathrm{NE}$ of the emerging technologies towards enabling Fifth Generation (5G) is heterogeneous networks (HetNets) which include Green Small Cell Networks consisting of lowpower base station (BS), (e.g., microcells, picocells, and femtocells), overlaid within the macrocell geographical area, deployed by either users or network operators who share the same spectrum with the macrocells [1] and [2]. The purpose of HetNets is to allow user equipments (UEs) to access small cells even though the UEs are within the coverage of macrocell. The deployment of small cells has a great potential to improve the spatial reuse of radio resources and also to enhance the energy efficiency (EE) of the network [2] and [3]. Although, some works [4] and [5] have been done on fairness based energy efficient radio resource management in traditional OFDMA systems mainly maximising either EE or spectral efficiency (SE). In [6], authors proposed a MOP approach to jointly maximise EE and SE along with fairness for downlink transmission scheme of the traditional OFDMA systems.

This work was partially supported by the UK EPSRC under Garnt number EP/K011693/1 and the EU FP7 under Grant Number PIRSES-GA-2013610524.
Most of the work in the literature mainly focuses on maximising EE or SE with respect to the transmission power without considering the backhaul energy consumption [7]. The authors in [8] proposed a mechanism to compute backhaul energy efficiency (BEE) in a heterogeneous network deployment consisting of a macrocell with enabled device to device (D2D) communication to reduce the overall network power consumption in comparison to the small cell deployment. In [9], the authors analysed the energy efficiency optimisation with subject to SE constraint in the downlink of Green HetNets using Coordinated Multi-Point (CoMP) transmission scheme to reduce the total power consumption including the backhaul power consumption for two backhauling technologies, i.e., microwave and fiber. The contribution of the backhaul energy consumption to the total energy consumption is dependent on the network deployment scenario and technology and the topology of the backhaul itself [10].

According to the best of our knowledge, there is no previous work on joint throughput and BEE tradeoff with fairness in downlink transmission scheme of two-tier HetNets considering multi-user multi-carrier systems. In this paper, we investigate the two conflicting objectives such as jointly maximising throughput and BEE subject to minimum QoS requirements, maximum input power constraint and rate fairness level $\omega$ as a multi-objective optimisation problem (MOP). The MOP is transformed into a single-objective optimisation problem (SOP) using weighted sum method obtaining a complete Pareto-optimal set or Pareto Frontier providing a quantitative insight into the throughput and BEE tradeoff with different rate fairness level $\omega$.

\section{System Model and Problem Formulation}

We consider a downlink scenario of two-tier HetNets consisting of a macrocell and $K-1$ pico BS's with the total number of users $M$ and $N$ non-overlapping subcarriers. We denote the index set of all subcarriers as $n=\{1, \cdots, N\}$, the set of all users as $m=\{1, \cdots, M\}$ and the set of networks as $k=\{1, \cdots, K\}$. Further, we consider an orthogonal subcarrier selection scheme which assigns each subcarrier exclusively to either pico BS (PB) or macrocell (Mc) at any time. We assume that $N_{\mathrm{k}}$ indicates the set of all subcarriers allocated to the network $k$ and $\left|N_{k}\right|$ is the cardinality of the set $N_{k}$ denoting the total number of subcarriers allocated to the network $k$. The instantaneous rate of user $m$ associated 
with network $k$ on subcarrier $n$ is given as follow:

$$
\mathrm{r}_{m, n}^{(\mathrm{k})}=\theta_{k} B_{k} \log _{2}\left(1+\gamma_{m, n}^{(\mathrm{k})} \times p_{m, n}^{(k)}\right),
$$

where $\theta_{k}$ is the proportion of bandwidth allocated to each subcarrier by network $k$ and is given by $\frac{1}{\left|N_{k}\right|} \cdot B_{k}$ indicates the total bandwidth available to the network $k$ and $p_{m, n}^{(\mathrm{k})}$ indicates the power allocated to the subcarrier $n$ for user $m$ in network $k$. The channel-to-noise-ratio (CNR) of user $m$ on subcarrier $n$ associated with network $k$ can be defined as $\gamma_{m, n}^{(\mathrm{k})}=\frac{g_{m, n}^{(\mathrm{k})}}{\rho_{m, n}^{(\mathrm{k})} N_{0} \theta_{k} B_{k}}$. The total data rate of user $m$ is

$$
R_{m}=\sum_{k=1}^{K} \sum_{n=1}^{N} \rho_{m, n}^{(k)} r_{m, n}^{(k)}
$$

where $\rho_{m, n}^{(k)}$ is the subcarrier allocation indicator such that $\rho_{m, n}^{(k)} \in\{0,1\}$. To model fairness, we adopt $\omega$-fair utility function

$$
u_{\omega}\left(R_{m}\right)= \begin{cases}\ln \left(R_{m}\right), & \text { if } \omega=1, \\ R_{m}^{1-\omega} /(1-\omega), & \text { if } \omega \neq 1, \omega \geq 0,\end{cases}
$$

where the value of $\omega$ represents different rate fairness levels. For no fairness requirement, $\omega=0$, and $u_{\omega}\left(R_{m}\right)=R_{m}$. By increasing $\omega$, the rate fairness among users also increases. For the special case of $\omega \rightarrow \infty$, an absolute rate fairness among users is achieved.

\section{A. Power Consumption Model for two-tier HetNets}

Hence, the overall consumed power in downlink of two-tier HetNets can be modelled as below:

$$
P=\epsilon_{0} \sum_{k=1}^{K} \sum_{n=1}^{N} \sum_{m=1}^{M} \rho_{m, n}^{(k)} p_{m, n}^{(k)}+K \times P_{\mathrm{C}}+P_{B H},
$$

where $P_{B H}$ is the backhaul power consumption. In two-tier HetNets, the backhaul power consumption consists of the backhaul power consumed at aggregation switch (or switches) $P_{B H}^{(m b)}$, to forward the traffic from all the macro BS's to the core network and the backhaul power consumed at sink switch (or switches) $P_{B H}^{(s c)}$, located at the macro BS to receive the traffic from the $K-1$ small cells then aggregate it with the macrocell BS traffic and forward it to the core network. Optical fiber is most commonly used for backhaul links between all macro BS's to the aggregation switch. The backhaul power consumption $P_{B H}^{(m b)}$ can be expressed as follow [11]:

$$
P_{B H}^{(m b)}=\left[\frac{I_{m b}}{\max _{d l}}\right] \times P_{s w}+I_{m b} \times P_{d l}+L_{u l} \times P_{u l},
$$

where $I_{m b}$ is the number of macro BS's which is equal to 1 according to our system model, $\max _{d l}$ is the maximum number of downlink interfaces at aggregation switch of macro BS and $P_{d l}$ is the power consumption of a downlink interface at the macro BS aggregation switch. $L_{u l}=\left(\frac{T_{a g g}}{C_{\max }}\right)$ and $P_{u l}$ are the total number of uplink interfaces and power consumption of an uplink interface, respectively. $T_{a g g}$ and $C_{\text {max }}$ are the total traffic at the aggregation switches of the macrocell BS and the maximum transmission rate of an uplink interface, respectively. $P_{s w}$ represents the power consumption of the aggregation switch at the macrocell BS and (5) can be rewritten as:

$$
\begin{aligned}
& P_{B H}^{(m b)}=\left[\frac{1}{\max _{d l}}\right]\left(\beta \cdot P_{s w}^{\max }+(1-\beta) \frac{C_{a g g}}{C_{s w}^{\max }} P_{s w}^{\max }\right) \\
& +P_{d l}+\left(\frac{T_{a g g}}{C_{\max }}\right) P_{u l},
\end{aligned}
$$

where $\beta \in[0,1], P_{s w}^{\max }$ is the maximum power consumption of the switch, $C_{a g g}$ is the total traffic at the macrocell aggregation switch and $C_{s w}^{\max }$ denotes the maximum traffic switch can handle.

Similarly, either optical fiber or microwave can be used for backhaul links between all the small cells and the sink switch located at the macro BS. However, in this paper we assume that optical fiber is used and $P_{B H}^{(s c)}$ can be defined as [9]:

$$
P_{B H-F i b e r}^{(s c)}=\left[\frac{K-1}{\max _{d l}}\right] P_{s w}+\left(\frac{C_{s}}{C_{\max }}\right) P_{u l},
$$

where $C_{s}$ denotes the total traffic of the small cells. Hence, $P_{B H}^{(s c)}$ can also be defined for the case where all the traffic from the small cells goes to the core network via internet without using aggregation node at macrocell as [8]:

$P_{B H}^{(s c)}=\left[\frac{(K-1) C_{s}}{4 G b p s}\right]\left[\frac{P_{\text {router }}}{40}+P_{\mathrm{OLT}}\right]+(K-1) P_{\mathrm{ONU}}$,

where $P_{\text {router }}$ represents the power consumption of the edge router, $P_{\mathrm{OLT}}$ denotes the power consumption of the OLT and $P_{\mathrm{ONU}}$ represents power consumption of ONU. The total power consumption of two-tier HetNets can be written as:

$$
P_{\text {total }}=\epsilon_{0} \sum_{k=1}^{K} P_{k}^{\max }+K \times P_{\mathrm{C}}+P_{B H}^{\max },
$$

where $P_{k}^{\max }$ is the maximum transmission power of network $k, P_{\mathrm{C}}^{k}$ is the circuit power of network $k$ and $P_{B H}^{\max }$ is the maximum power consumed by the backhaul to forward the collected traffic (i.e., when all networks are operating at their maximum transmission power) to the core network. Now, we can define the Backhaul Energy Efficiency (BEE) as follow:

$$
\mathrm{BEE}=\frac{\sum_{m=1}^{M} R_{m}}{\epsilon_{0} \sum_{k=1}^{K} \sum_{n=1}^{N} \sum_{m=1}^{M} \rho_{m, n}^{(k)} p_{m, n}^{(k)}+K \times P_{\mathrm{C}}+P_{B H}}
$$

Similalrly, we can also define Energy Efficiency (EE) as a special case of (10) when no backhaul power consumption is assumed, i.e., $P_{B H}=0$.

\section{B. Problem Formulation}

Our goal is to simultaneously optimise throughput and BEE with fairness and QoS guarantees while ensuring that the interference power does not exceed their specific thresholds. The joint optimisation problem to maximise the throughput and $\mathrm{BEE}$ is equivalent to maximising the sum rate and minimising the total power consumption. In this section, we investigate the Throughput-BEE tradeoff in downlink transmission scheme of two-tier HetNets as a multi-objective optimization problem 
(MOP) by normalising the two conflicting objective functions to ensure a consistent comparison as below:

$$
\begin{aligned}
& \text { (P1) } \max _{\mathbf{p}, \boldsymbol{\rho}} \frac{\left(\sum_{m=1}^{M} u_{\omega}\left(R_{m}\right)-u_{\omega}^{\min }\right)}{\left(u_{\omega}^{\max }-u_{\omega}^{\min }\right)} \text { and } \max _{\mathbf{p}, \boldsymbol{\rho}} \frac{-P}{P_{\text {total }}} \text {, } \\
& \text { s.t. } \mathrm{C} 1: \sum_{m=1}^{M} \sum_{n=1}^{N} \rho_{m, n}^{(k)} p_{m, n}^{(k)} \leq P_{k}^{\max }, \forall k \text {. } \\
& \mathrm{C} 2: \quad R_{m} \geq R_{m}^{\min }, \forall m \text {. } \\
& \text { C3 : } \quad p_{m, n}^{(k)} \geq 0, \forall m, \forall n, \forall k \text {. } \\
& \mathrm{C} 4: \quad \sum_{m=1}^{M_{k}} \rho_{m, n}^{(k)} \leq 1, \quad \forall n, k \text {. } \\
& \text { C5 : } \quad \rho_{m, n}^{(k)} \in[0,1], \forall m, \forall n, \forall k .
\end{aligned}
$$

where $u_{\omega}^{\max }$ are the maximum achievable utility value of (3) for a given value of $\omega$ under the constraints C1-C5. $u_{\omega}^{\min }$ is the minimum achievable utility value computed by setting $R_{m}=\delta$ in (3) for a given value of $\omega$ where $\delta>0$ is a predefined sufficiently small value. $P_{k}^{\max }$ is the maximum transmission power of network $k$ and $R_{m}^{\min }$ is the minimum rate requirement for each user $m$. $\mathrm{C} 1$ is the maximum transmission power of each network $k$ which should not exceed $P_{k}^{\max }$. $\mathrm{C} 2$ is the minimum rate requirement for each user which is applicable only if user $m$ is admitted, i.e., $\rho_{m, n}^{(k)}=1$. C3 ensures that the power $p_{m, n}^{(k)}$ should be positive. C4 and C5 indicate that $\rho_{m, n}^{(k)}$ is a binary variable such that each subcarrier $n$ can be exclusively assigned to one user within network $k$. For better tractability, we relax the constraint C6 by allowing time sharing.

The MOP defined in (11) can be transformed into a single objective optimization problem (SOP) by applying the weighted sum method [12] as follow:

$$
\begin{gathered}
\text { (P2) } \max _{\mathbf{p}, \boldsymbol{\rho}} \alpha \frac{\sum_{m=1}^{M} u_{\omega}\left(R_{m}\right)-u_{\omega}^{\min }}{u_{\omega}^{\max }-u_{\omega}^{\min }}-(1-\alpha) \frac{P}{P_{\text {total }}}, \\
\text { s.t. } \\
\mathrm{C} 1-\mathrm{C} 5
\end{gathered}
$$

where $\alpha \in[0,1]$ is the Throughput-BEE tradeoff biasing factor.

\section{Proposed Distributed Solution}

In this section, we propose a distributed solution to the problem (P2) for different values of $w$ which can collectively form the Pareto optimal set. In other words, by tuning $\alpha$, we investigate the Throughput-BEE tradeoff for a given value of $\omega$.

Firstly for the case of $\omega>0$, we define a vector $\mathbf{x}=$ $\left[x_{1}, x_{2}, \ldots, x_{m}\right]^{T}$ and rewrite $(\mathbf{P} 2)$ as

(P3)

$$
\begin{gathered}
\max _{\mathbf{x}, \mathbf{p}, \boldsymbol{\rho}} \alpha \frac{\sum_{m=1}^{M} u_{\omega}\left(x_{m}\right)-u_{\omega}^{\min }}{u_{\omega}^{\max }-u_{\omega}^{\min }}-(1-\alpha) \frac{P}{P_{\text {total }}} \\
\text { s.t. } \mathrm{C} 1-\mathrm{C} 5 \\
\mathrm{C} 6: x_{m} \leq R_{m}, \forall m .
\end{gathered}
$$

where $\mathbf{p}=\left\{p^{(1)}, p^{(2)}, \cdots, p^{(K)}\right\}$ and $\boldsymbol{\rho}=$ $\left\{\rho^{(1)}, \rho^{(2)}, \cdots, \rho^{(K)}\right\}$. It is worthwhile to mention that $p^{(1)}, p^{(2)}$ and $p^{(K)}$ are $M \times N$ power allocation indication matrix. The size of $\rho^{(1)}, \rho^{(2)}$ and $\rho^{(K)}$ are also same as $p^{(1)}, p^{(2)}$ and $p^{(K)} \cdot u_{\omega}(\cdot)$, is a strictly increasing function, hence, for an optimal solution, $x_{m}$ must be equal to $R_{m}$.

We then utilize Hierarchical Decomposition method [13] to find an optimal solution to (P3). To characterize the duality gap between the primal and dual solutions, the time-sharing condition is first defined in [14] and it is proved that if it holds, the duality gap is zero even if the original optimization problem is not convex. In practical multicarrier systems with a large number of subcarriers, channel conditions in adjacent subcarriers are often similar. In such case, the time-sharing condition is approximately satisfied, and accordingly the duality gap is nearly zero [14]. We define the partial Lagrangian function of primal problem in (P3) formed by dualising the constraint C6:

$$
\begin{aligned}
L(\mathbf{p}, \boldsymbol{\rho}, \mathbf{x}, \lambda) & =\alpha \frac{\sum_{m=1}^{M} u_{\omega}\left(x_{m}\right)-u_{\omega}^{\min }}{u_{\omega}^{\max }-u_{\omega}^{\min }}-(1-\alpha) \frac{P}{P_{\text {total }}} \\
& +\sum_{m=1}^{M} \lambda_{m}\left(R_{m}-x_{m}\right) \\
= & \left(\begin{array}{c}
\left.\alpha \frac{\sum_{m=1}^{M} u_{\omega}\left(x_{m}\right)-u_{\omega}^{\min }}{u_{\omega}^{\max }-u_{\omega}^{\min }}-\sum_{m=1}^{M} \lambda_{m} x_{m}\right) \\
+\left(\sum_{m=1}^{M} \lambda_{m} R_{m}-(1-\alpha) \frac{P}{P_{\text {total }}}\right)
\end{array}\right.
\end{aligned}
$$

where $\boldsymbol{\lambda}=\left[\lambda_{1}, \lambda_{2}, \ldots, \lambda_{M}\right]^{T}$ is the dual vector for constraint C6 corresponding to each user. Then the dual function is

$$
g(\boldsymbol{\lambda})=\left\{\begin{array}{l}
\max _{\mathbf{x}, \mathbf{p}, \boldsymbol{\rho}} L(\mathbf{p}, \boldsymbol{\rho}, \mathbf{x}, \boldsymbol{\lambda}) \\
\text { s.t. } \mathrm{C} 1-\mathrm{C} 5
\end{array}\right.
$$

Obviously, the dual function in (15) can be separated into two maximisation subproblems as shown in (16) and (17) respectively.

$$
\begin{aligned}
& g_{1}(\boldsymbol{\lambda})=\max _{\mathbf{x}} f(\mathbf{x})=\alpha \frac{\sum_{m=1}^{M} u_{\omega}\left(x_{m}\right)-u_{\omega}^{\min }}{u_{\omega}^{\max }-u_{\omega}^{\min }}-\sum_{m=1}^{M} \lambda_{m} x_{m}, \\
& g_{2}(\boldsymbol{\lambda})=\left\{\begin{array}{c}
\max _{\mathbf{p}, \boldsymbol{\rho}} \sum_{m=1}^{M} \lambda_{m} R_{m}-(1-\alpha) \frac{P}{P_{\text {total }}}, \\
\text { s.t. } \mathrm{C} 1-\mathrm{C} 5 .
\end{array}\right.
\end{aligned}
$$

\section{A. Solution to subproblem $g_{1}(\boldsymbol{\lambda})$ :}

In (16), as $u_{\omega}\left(x_{m}\right)$ is a concave function of $x_{m}$ and hence, $f(\mathbf{x})$ is also a concave function of $x_{m}$. Therefore, the optimality of (16) can be solved by taking the derivative of $f(\mathbf{x})$ with respect to $x_{m}$ and setting it equal to zero as given by

$$
x_{m}^{*}=\left[\sqrt[\omega]{\frac{\alpha}{\lambda_{m}\left(u_{\omega}^{\max }-u_{\omega}^{\min }\right)}}\right]^{+}, \forall m
$$

where $(y)^{+} \triangleq \max (0, y)$ and $x_{m}^{*}$ is the value of $x_{m}$ which maximises (16).

The corresponding dual problem is

$$
\min _{\boldsymbol{\lambda} \geq 0} g(\boldsymbol{\lambda}) .
$$


The dual problem (19) can be solved using subgradient method [14]. The dual vector $\lambda$ can be updated as follow:

$$
\lambda_{m}(i+1)=\left[\lambda_{m}(i)-s_{0}\left(R_{m}-x_{m}\right)\right]^{+}, \forall m
$$

where $s_{0}$ is the positive step size.

\section{B. Solution to subproblem $g_{2}(\boldsymbol{\lambda})$ :}

The subproblem (17) can be solved using Lagrangian dual decomposition method [13]. By relaxing the constraints $\mathrm{C} 1$ $\mathrm{C} 2$, the Lagrangian function becomes

$$
\begin{aligned}
& T(\mathbf{p}, \boldsymbol{\rho}, \mu, \eta)=\sum_{m=1}^{M} \lambda_{m} R_{m}-(1-\alpha) \frac{P}{P_{\text {total }}}+\sum_{k=1}^{K} \mu_{k} \\
& \left(P_{k}^{\max }-\sum_{n=1}^{N} \sum_{m=1}^{M} \rho_{m, n}^{(k)} p_{m, n}^{(k)}\right)+\sum_{m=1}^{M} \eta_{m}\left(R_{m}-R_{m}^{\min }\right) \\
& =\sum_{n=1}^{N}\left[\sum_{k=1}^{K} \sum_{m=1}^{M}\left(\left(\lambda_{m}+\eta_{m}\right) r_{m, n}^{(k)}-\left(\frac{(1-\alpha) \varepsilon_{0}}{P_{\text {total }}}\right) \times p_{m, n}^{(k)}\right)\right. \\
& \left.\rho_{m, n}^{(k)}\right]+\sum_{k=1}^{K} \mu_{k} P_{k}^{\max }-\frac{(1-\alpha)\left(P_{\mathrm{C}}+P_{\mathrm{BH}}\right)}{P_{\text {total }}}-\sum_{m=1}^{M} \eta_{m} R_{m}^{\min },
\end{aligned}
$$

where $\boldsymbol{\mu}=\left[\mu_{1}, \mu_{2}, \ldots, \mu_{K}\right]^{T}$ and $\boldsymbol{\eta}=\left[\eta_{1}, \eta_{2}, \ldots, \eta_{M}\right]^{T}$ are the dual vectors corresponding to the constraints $\mathrm{C} 1$ and $\mathrm{C} 2$. We further observe that the dual function

$$
h(\mu, \eta)=\left\{\begin{array}{c}
\max _{\mathbf{p}, \boldsymbol{\rho}} T(\mathbf{p}, \boldsymbol{\rho}, \mu, \eta), \\
\text { s.t. C3, C4 and C5 }
\end{array}\right.
$$

can be decoupled into $N$ subproblems, which can be independently solved for each subcarrier $n$. The subproblem corresponding to subcarrier $n$ at given $(\mu, \eta)$ is

$$
\begin{aligned}
T_{n}(\mu, \eta)=\max _{\mathbf{p}(:, \mathbf{n}), \boldsymbol{\rho}(:, \boldsymbol{n})}\left[\sum _ { k = 1 } ^ { K } \sum _ { m = 1 } ^ { M } \left(\left(\lambda_{m}+\eta_{m}\right) r_{m, n}^{(k)}\right.\right. \\
\left.\left.-\left(\frac{(1-\alpha) \varepsilon_{0}}{P_{\text {total }}}+\mu_{k}\right) p_{m, n}^{(k)}\right) \rho_{m, n}^{(k)}\right], \\
\text { subject to } \mathrm{C} 3-\mathrm{C} 5,
\end{aligned}
$$

where $\mathbf{p}(:, \mathbf{n})$ and $\boldsymbol{\rho}(:, \boldsymbol{n})$ are the matrix of $p_{m, n}^{(k)}$ and $\rho_{m, n}^{(k)}$ at subcarrier $n$ respectively. Due to the constraints $\mathrm{C} 4$ and $\mathrm{C} 5$, the subcarrier allocation indicator $\rho(:, n)$ is an all-zero matrix except for one binary non-zero entry. Hence, for a certain subcarrier $n$, we can calculate $C_{m, n}^{(k)}$ for each user $m$ associated with network $k$ as

$$
C_{m, n}^{(k)}=\left\{\begin{array}{l}
\max _{\mathbf{p}(:, \mathbf{n})}\left(\left(\lambda_{m}+\eta_{m}\right) r_{m, n}^{(k)}-X p_{m, n}^{(k)}\right) \\
\text { s.t. } p_{m, n}^{(k)} \geq 0, \quad \forall m, \forall k
\end{array}\right.
$$

where $X=\left(\frac{(1-\alpha) \varepsilon_{0}}{P_{\text {total }}}+\mu_{k}\right)$. Therefore, we calculate the optimal value of (23) at given $\boldsymbol{\lambda}, \boldsymbol{\mu}$ and $\boldsymbol{\eta}$ to determine the subcarrier assignment indicator for subcarrier $n$ as

$$
\rho_{m, n}^{(k)}= \begin{cases}1, & \text { if }\left(m^{*}, k^{*}\right)=\arg \max _{m, k} C_{m, n}^{(k)}, \forall n . \\ 0, & \text { otherwise. }\end{cases}
$$

Then, by using the KKT conditions for a fixed set of Lagrange multipliers, an optimal power allocation to user $m$ associated with network $k$ on subcarrier $n$ is obtained as

$$
p_{m, n}^{(k)}=\left[\frac{\left(\lambda_{m}+\eta_{m}\right) \theta_{k} B_{k}}{\left(\frac{(1-\alpha) \varepsilon_{0}}{P_{\text {total }}}+\mu_{k}\right) \ln 2}-\frac{1}{\gamma_{m, n}^{(k)}}\right]^{+} .
$$

Once all $N$ subproblems in (19) are solved, $h(\mu, \eta)$ is derived by (17) and (19) at given $(\mu, \eta)$. The subproblem in (13) can be solved via the dual problem as given below

$$
\min _{\mu \geq 0, \eta \geq 0} h(\mu, \eta) \text {. }
$$

In order to solve the dual problem (26), the subgradient method can be used to update the dual vectors $\mu$ and $\eta$ in each iteration. The subgradient of $h(\mu, \eta)$ at the $i+1^{t h}$ iteration are given by

$$
\begin{gathered}
\mu_{k}(i+1)=\left[\mu_{k}(i)-s_{1}\left(P_{k}^{\max }-\sum_{n=1}^{N} \sum_{m=1}^{M} p_{m, n}^{(k)}\right)\right]^{+}, \forall k \\
\eta_{m}(i+1)=\left[\eta_{m}(i)-s_{2}\left(R_{m}-R_{m}^{\mathrm{min}}\right)\right]^{+}, \forall m
\end{gathered}
$$

where $s_{j}, j \in\{1,2\}$ are the positive step sizes.

Finally for the case of $\omega=0$, i.e., $u_{\omega}\left(R_{m}\right)=R_{m}$, (P2) can be solved directly using dual decomposition method (similar to the solution to subproblem $g_{2}(\lambda)$ ). The optimal power allocation to user $m$ associated with network $k$ on subcarrier $n$ is given by

$$
p_{m, n}^{(k)}=\left[\frac{\left(\frac{\alpha}{u_{0}^{\max }-u_{0}^{\min }}+\eta_{m}\right) \theta_{k} B_{k}}{\left(\frac{(1-\alpha) \varepsilon_{0}}{P_{\text {total }}}+\mu_{k}\right) \ln 2}-\frac{1}{\gamma_{m, n}^{(k)}}\right]^{+},
$$

Similarly, for a certain subcarrier $n$, we can calculate $D_{m, n}^{(k)}$ for each user $m$ associated with network $k$ as

$$
D_{m, n}^{(k)}=\left\{\begin{array}{l}
\max _{\mathbf{p}(:, \mathbf{n})}\left(\left(\frac{\alpha}{u_{0}^{\max }-u_{0}^{\min }}+\eta_{m}\right) r_{m, n}^{(k)}-X p_{m, n}^{(k)}\right) \\
\text { s.t. } p_{m, n}^{(k)} \geq 0, \quad \forall m, \forall k .
\end{array}\right.
$$

The subcarrier assignment indicator for subcarrier $n$ as

$$
\rho_{m, n}^{(k)}=\left\{\begin{array}{l}
1, \text { if }\left(m^{*}, k^{*}\right)=\arg \max _{m, k} D_{m, n}^{(k)}, \\
0, \text { otherwise. }
\end{array}\right.
$$

\section{Simulation Results}

In the simulations, we consider a two-tier HetNets consisting of a macrocell overlaid with $K-1$ small cells with $M$ users being randomly distributed and $N$ subcarriers. More details about the simulation parameters can be found in [8] [9] and are mentioned in Table. I.

Fig. 1 investigates the impact of weighting coefficient $\alpha$ on the achievable EE and throughput for various values of $\omega$. At $\alpha=0$, the proposed MOP is transformed into minimising the total consumption power whereas at $\alpha=1$ it is transformed into maximising throughput. As it can be seen from Fig. 1, achievable EE and throughput can be varied by adjusting the 
TABLE I: Simulation Parameters.

\begin{tabular}{|c|c||c|c|}
\hline Parameter & Value & Parameter & Value \\
\hline $\mathrm{N}$ & 256 & $\mathrm{~K}$ & 5 \\
\hline $\mathrm{M}$ & 8 & $\mathrm{~B}[\mathrm{MHz}]$ & 3 \\
\hline$P_{\mathrm{C}}[\mathrm{W}]$ & 0.4 & $\epsilon_{0}$ & $38 \%$ \\
\hline$P_{\operatorname{macro}}^{\max }[\mathrm{W}]$ & 40 & $P_{\text {small }}^{\max }[\mathrm{W}]$ & 0.2 \\
\hline$N_{0}[\mathrm{dBM} / \mathrm{Hz}]$ & -174 & $R_{\text {macro }}[\mathrm{m}]$ & 500 \\
\hline$R_{\text {small }}[\mathrm{m}]$ & 100 & Log-Normal Shadowing & $N(0, \sigma)$ \\
\hline std dev $\sigma[\mathrm{dB}]$ & 8 & $R_{m}^{\min }[\mathrm{Kbps}]$ & 500 \\
\hline $\max _{d l}$ & 24 & $P_{s w}^{\max }[\mathrm{W}]$ & 300 \\
\hline$P_{d l}[\mathrm{~W}]$ & 1 & $P_{u l}[\mathrm{~W}]$ & 2 \\
\hline$C_{\max }[\mathrm{Gbps}]$ & 10 & $\beta$ & 0.9 \\
\hline$C_{s w}^{\max }[\mathrm{Gbps}]$ & 24 & $P_{\text {router }}[\mathrm{kW}]$ & 4 \\
\hline$P_{\mathrm{ONU}}[\mathrm{W}]$ & 4.69 & $P_{\mathrm{OLT}}[\mathrm{W}]$ & 100 \\
\hline & & &
\end{tabular}

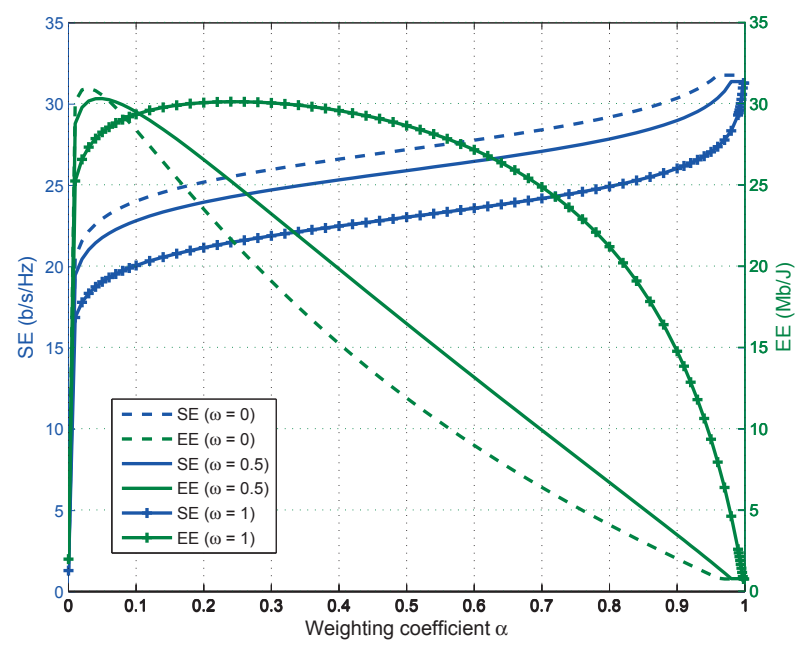

Fig. 1: $\mathrm{EE}$ and $\mathrm{SE}$ versus $\alpha$ for different values of $\omega$.

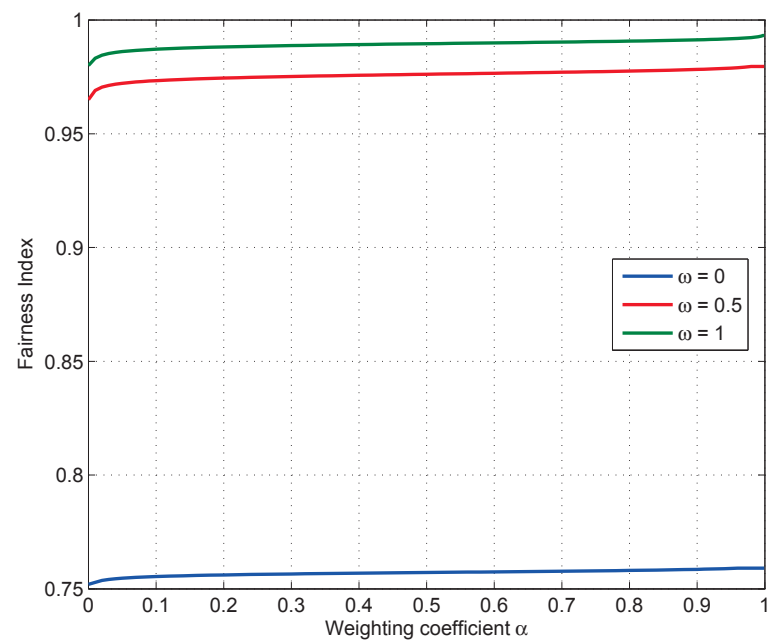

Fig. 2: Fairness Index versus $\alpha$ for different values of $\omega$.

value of $\omega$. For example, at $\alpha=0$ and $\omega=0$, an achievable throughput and EE are $1.358 \mathrm{~b} / \mathrm{s} / \mathrm{Hz}$ and $2.037 \mathrm{Mb} / \mathrm{J}$, respectively whereas at $\alpha=0$ and $\omega=1$, an achievable Throughput and $\mathrm{EE}$ are $1.311 \mathrm{~b} / \mathrm{s} / \mathrm{Hz}$ and $1.967 \mathrm{Mb} / \mathrm{J}$, respectively. We further observe that an achievable EE gradually increases with $\alpha$ to an optimal EE, and then afterwards starts decreasing with an increase in $\alpha$. Similarly, an achievable SE always increases

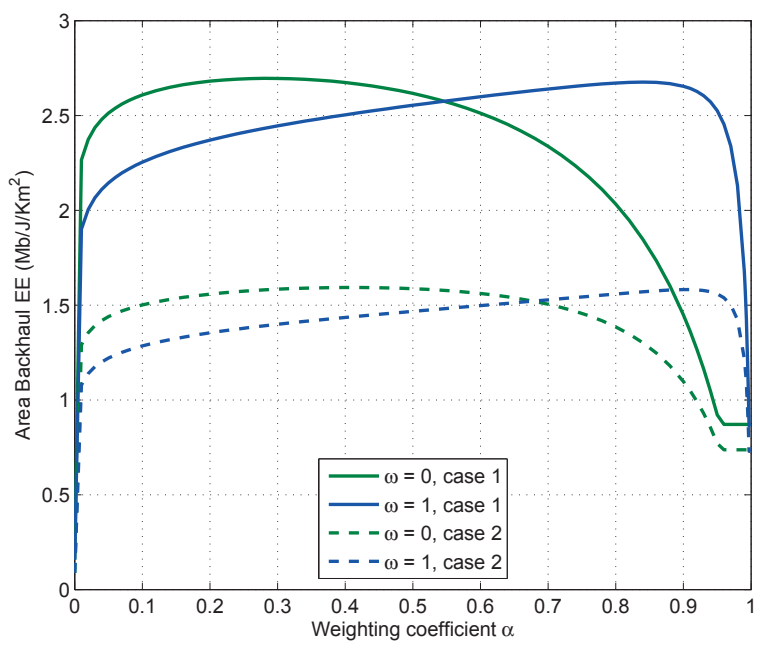

Fig. 3: Area BEE versus $\alpha$ for various values of $\omega$.

with an increase in $\alpha$ and on the other hand, an achievable SE always decreases with an increase in $\omega$. One of the main observation is that an optimal EE decreases with an increase in $\omega$, due to the fact that the higher level of fairness is achieved at the cost of degradation in achievable EE. It is also worthwhile to mention that an optimal $\mathrm{EE}$ at smaller value of $\omega$ results in higher achievable Throughput. Nevertheless, the proposed MOP approach achieves the entire Pareto Frontier or complete Pareto optimal set of the proposed problem with different rate fairness levels $\omega$ and weighting coefficient $\alpha$.

In order to evaluate the fairness of the users in two-tier HetNets, we define the Jain's fairness index (FI) similar to [15] as below:

$$
\text { Fairness Index }(\mathrm{FI})=\frac{\left(\sum_{m=1}^{M}\left(\frac{R_{m}}{R_{m}^{\min }}\right)\right)^{2}}{K\left(\sum_{m=1}^{M}\left(\frac{R_{m}}{R_{m}^{\text {min }}}\right)^{2}\right)} \text {. }
$$

Fig. 2 shows the fairness index among all the users in a single macrocell overlaid with $K-1$ small cells versus weighting coefficient $\alpha$ for various values of $\omega$. It is quite obvious from the figure, highest fairness index is achieved at $\omega=1$ whereas the lowest fairness index is achieved when no fairness is considered, i.e., $\omega=0$. It can be easily observed that the higher fairness index is achieved at the expense of reduction in the throughput.

Fig. 3 shows the impact of the weighting coefficient $\alpha$ on the normalised BEE over the total coverage area for various values of rate fairness level $\omega$. In Fig. 3, the BEE comparison in two-tier HetNets is shown for the two different cases of backhauling technologies. In case 1, an optical fiber is used as a technology to backhaul traffic from a macrocell to the aggregation switch (can be one or more) and all the traffic from $K-1$ small cells are backhauled via Internet without going through the aggregation switch at the macrocell. In case 2, an optical fiber is used as a technology to backhaul traffic from a macrocell to the aggregation switch (one or more) and all the traffic from $K-1$ small cells is collected at the sink node of the macrocell and backhauled from macrocell to the core network using an optical fiber link. One of the intuition from the figure 


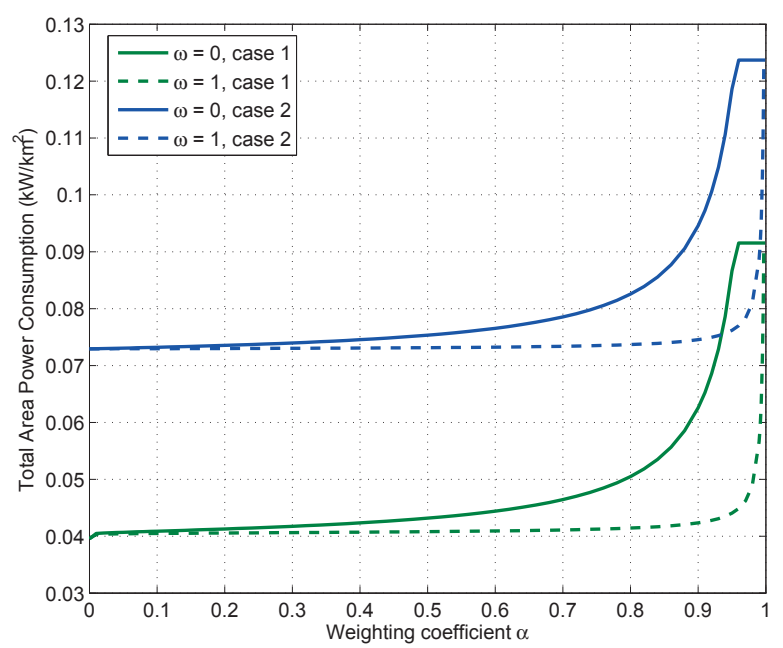

Fig. 4: Total Area Power Consumption versus $\alpha$ for various values of $\omega$.

is that BEE first increases with weighting coefficient $\alpha$ until an optimal value of $\alpha$ and afterwards it starts decreasing with weighting coefficient $\alpha$. At the lower values of $\omega$, an optimal BEE is achieved at lower values of $\alpha$ whereas an optimal BEE is achieved at higher values of $\alpha$ for the case of the higher values of $\omega$.

Fig. 4 shows the impact of two backhauling technologies on the total area power consumption of two-tier HetNets versus weighting coefficient $\alpha$ for various values of rate fairness level $\omega$. The total area power consumption increases with an increase in $\alpha$ whereas it decreases with an increase in $\omega$. It is quite obvious from the figure that at a given value of $\omega$, the total area power consumption in case 1 is always less than case 2 at the expense of degradation in area BEE as shown in Fig 3. At the values of $\omega=0$ and $\alpha=1$, the total area power consumption in two-tier HetNets without backhaul power consumption is $0.05 \mathrm{~kW} / \mathrm{km}^{2}$ as compared to 0.092 $\mathrm{kW} / \mathrm{km}^{2}$ and $0.125 \mathrm{~kW} / \mathrm{km}^{2}$ for case 1 and case 2 , respectively. This affirms the observation that the impact of backhaul power consumption in two-tier HetNets is larger than to the case where no backhaul power consumption is assumed irrespective of the used backhauling technology. By dynamically choosing a higher value of $\omega$, the total area power consumption can be reduced for all values of $\alpha$ irrespective of the used backhauling technology.

\section{CONCLUSION}

In this paper, we utilise the concept of MOP to jointly optimise the throughput and BEE tradeoff in two-tier HetNets with QoS and fairness guarantee constraints. The complete Pareto optimal set is obtained by employing the weighted sum method to transform our proposed MOP into an SOP which can be solved using Lagrangian Dual Decomposition (LDD) method. We further investigate the impact of rate fairness level $\omega$, interference threshold $I_{n}^{\text {th }}$ and weighting coefficient $\alpha$ on achievable throughput and $\mathrm{EE}$ with or without backhaul power consumption. The network operators can have more flexibility to satisfy the user's QoS requirements along with reducing their total area power consumption by dynamically tuning the weighting coefficient $\alpha$ and rate fairness level $\omega$.

\section{REFERENCES}

[1] Q. Ye, B. Rong, Y. Chen, M. Al-Shalash, C. Caramanis, and J. Andrews, "User association for load balancing in heterogeneous cellular networks," IEEE Trans. on Wireless Commun.,, vol. 12, no. 6, pp. 27062716, June 2013.

[2] H. Pervaiz, L. Musavian, and Q. Ni, "Joint user association and energyefficient resource allocation with minimum-rate constraints in two-tier hetnets," in Proc. IEEE Int. Sym. on Personal Indoor and Mobile Radio Communications (PIMRC), Sept. 2013, pp. 1634-1639.

[3] R. Hernandez-Aquino, D. McLernon, M. Ghogho, and S. Zaidi, "Energy efficiency in MIMO large scale two-tier networks with beamforming and adaptive modulation," in Proc. 21st European Signal Processing Conference (EUSIPCO), Sept 2013, pp. 1-5.

[4] C. He, G. Li, F.-C. Zheng, and X. You, "Energy-efficient resource allocation in OFDM systems with distributed antennas," IEEE Trans. on Veh. Technol., vol. 63, no. 3, pp. 1223-1231, March 2014.

[5] Z. Ren, S. Chen, B. Hu, and W. Ma, "Energy-efficient resource allocation in downlink ofdm wireless systems with proportional rate constraints," IEEE Trans. on Veh. Technol., vol. 63, no. 5, pp. 2139-2150, Jun 2014.

[6] Z. Song, Q. Ni, K. Navaie, S. Hou, and S. Wu, "Energy and spectral efficiency tradeoff with $\alpha$-fairness in downlink OFDMA systems," IEEE Commun. Lett., vol. 19, no. 7, pp. 1265-1268, July 2015.

[7] C. Zarakovitis and Q. Ni, "Maximising energy efficiency in multiuser multicarrier broadband wireless systems: Convex relaxation and global optimisation techniques," IEEE Trans. on Vehicu. Tech., July 2015,DOI: 10.1109/TVT.2015.2455536.

[8] Y. Sambo, M. Shakir, K. Qaraqe, E. Serpedin, M. Imran, and B. Ahmed, "Energy efficiency improvements in HetNets by exploiting device-todevice communications," in Proc. 22nd European Signal Processing Conference (EUSIPCO), Sept 2014, pp. 151-155.

[9] K. Huq, S. Mumtaz, J. Bachmatiuk, J. Rodriguez, X. Wang, and R. Aguiar, "Green HetNet CoMP: Energy efficiency analysis and optimization," IEEE Trans. on Veh. Technol., vol. PP, no. 99, pp. 1-1, Nov. 2014.

[10] P. Monti, S. Tombaz, L. Wosinska, and J. Zander, "Mobile backhaul in heterogeneous network deployments: Technology options and power consumption," in Proc. 14th Int. Conf. on Transparent Optical Networks (ICTON), July 2012, pp. 1-7.

[11] S. Tombaz, P. Monti, K. Wang, A. Vastberg, M. Forzati, and J. Zander, "Impact of backhauling power consumption on the deployment of heterogeneous mobile networks," in Proc. IEEE Global Telecommunications Conference (GLOBECOM 2011), Dec 2011, pp. 1-5.

[12] R. T. Marler and J. S. Arora, "Survey of multi-objective optimization methods for engineering," Structural and multidisciplinary optimization, vol. 26, no. 6, pp. 369-395, 2004.

[13] D. P. Palomar and M. Chiang, "A tutorial on decomposition methods for network utility maximization," IEEE J. on Sel. Areas in Commun., vol. 24, no. 8, pp. 1439-1451, 2006.

[14] W. Yu and R. Lui, "Dual methods for nonconvex spectrum optimization of multicarrier systems," IEEE Trans. on Commun., vol. 54, no. 7, pp. 1310-1322, July 2006.

[15] T.-D. Nguyen and Y. Han, "A proportional fairness algorithm with QoS provision in downlink OFDMA systems," IEEE Commun. Lett., vol. 10, no. 11 , pp. 760-762, November 2006 